\title{
Cell Versus Chemokine Therapy Effects on Cell Mobilization to Chronically Dysfunctional Urinary Sphincters of Nonhuman
}

\section{Primates}

\author{
J. Koudy Williams ${ }^{1}$, Silmi Mariya ${ }^{2}$, Irma Suparto ${ }^{2}$, Shannon S. Lankford ${ }^{1}$, Karl-Erik Andersson ${ }^{1}$ \\ ${ }^{1}$ Wake Forest Institute for Regenerative Medicine, Winston-Salem, NC, USA \\ ${ }^{2}$ Primate Research Center Bogor Agricultural University, Bogor, Indonesia
}

Purpose: A major question remaining in approaches to tissue engineering and organ replacement is the role of native mobilized native cells in the regeneration process of damaged tissues and organs. The goal of this study was to compare the cell mobilizing effects of the chemokine CXCL12 and cell therapy on the urinary sphincter of nonhuman primates (NHP) with chronic intrinsic urinary sphincter dysfunction.

Methods: Either autologous lenti-M-cherry labeled skeletal muscle precursor cells (skMPCs) or CXCL12 were injected directly into the sphincter complex of female NHPs with or without surgery-induced chronic urinary sphincter dysfunction ( $n=4 /$ treatment condition). All monkeys had partial bone marrow transplantation with autologous lenti-green fluorescent protein (GFP) bone marrow cells prior to treatment. Labeled cells were identified, characterized and quantified using computer-assisted immunohistochemistry 6 months posttreatment.

Results: GFP-labeled bone marrow cells (BMCs) were identified in the bone marrow and both BMCs and skMPCs were found in the urinary sphincter at 6-month postinjection. BMCs and skMPCs were present in the striated muscle, smooth muscle, and lamina propria/urothelium of the sphincter tissue. Sphincter injury increased the sphincter content of BMCs when analyzed 6-month postinjection. CXCL12 treatment, but not skMPCs, increased the number of BMCs in all layers of the sphincter complex $(\mathrm{P}<0.05)$. CXCL12 only modestly $(\mathrm{P}=0.15)$ increased the number of skMPCs in the sphincter complex.

Conclusions: This dual labeling methodology now provides us with the tools to measure the relative number of locally injected cells versus bone marrow transplanted cells. The results of this study suggest that CXCL12 promotes mobilization of cells to the sphincter, which may contribute more to sphincter regeneration than injected cells.

Keywords: Lentivirus transduction; Urinary sphincter; Cell mobilization; Stem cells; Chemokines

- Grant/Fund Support: Funding for this study was provided by: R01 DK 083688 "Regeneration of the Lower Urinary Tract in Nonhuman Primates."

- Research Ethics: Studies in these nonhuman primates were approved by the Wake Forest University Institutional Animal Care and Use Committee, and were performed in compliance with the Animal Welfare Act and the Guide for the Care and Use of Laboratory Animals. Euthanasia was preformed according to the standards of the American Veterinary Medical Association.

- Conflict of Interest: Patent/intellectual property: Dr. Williams has a patent pending as follows: Pub. No.: US 2017/0106050 Al / Pub. Date: Apr. 20, 2017 / "Methods of Treating Incontinence and Other Sphincter Deficiency Disorders." Except for that, no potential conflict of interest relevant to this article was reported.

\section{- HIGHLIGHT}

- Chemokine therapy was superior to cell therapy in mobilizing cells to the urinary sphincter of nonhuman primates with chronic urinary dysfunction.

Corresponding author: J. Koudy Williams (iD https://orcid.org/0000-0002-4610-6412 Wake Forest Institute for Regenerative Medicine, Wake Forest University, 391 Technology Way Winston-Salem, NC, USA

E-mail: kwilliam@wakehealth.edu / Tel: +1-336-713-1323 / Fax: +1-336-713-7290

Submitted: June 7, 2018 / Accepted after revision: October 12, 2018
This is an Open Access article distributed under the terms of the Creative Commons Attribution Non-Commercial License (http://creativecommons.org/licenses/by-nc/4.0/) which permits unrestricted non-commercial use, distribution, and reproduction in any medium, provided the original work is properly cited. 


\section{INTRODUCTION}

The goal of regenerative medicine, including stem cell therapy, is to replace damaged or aged cells with healthy functioning cells in congenital defects, tissue injuries, autoimmune disorders, and neurogenic degenerative diseases.

One of the current challenges of regenerative medicine is to identify and track cells once they are injected, or implanted. The reason for this interest is to better understand the fate - and thus the potential role of these cells in the regeneration process. The field has evolved from the original thought that the primary role of implanted/injected cells is to incorporate and contribute to the main bulk of the regenerative process, to the current thought that regeneration is some combination of injected cell incorporation and stimulation of cell mobilization. If so, then the efficacy of these treatments would be dictated by the summation of these effects. Several factors have been shown to be important determinates of efficacy [1], and a tremendous amount of work has been done to optimize the efficacy of cell therapies [2]. Regardless of the source, route, dose or manipulation of the cells, a critical remaining question in regenerative medicine is the role of injected cells in tissue regeneration. Do cells contribute to tissue regeneration by direct integration, or do they primarily stimulate mobilization? Several approaches have been developed to follow or track cells once injected [3,4], including magnetic resonance and different reporting probes [2-4]. However, it remains difficult to determine the role of migrating cells to tissue regeneration.

We have previously found that local autologous skeletal muscle precursor cell (skMPC) therapy is not as effective in monkeys with chronic surgically-induced intrinsic sphincter deficiency (ISD) as it is in acute ISD [5]. However, the cell-produced, cellmobilizing chemokine CXCL12 is very effective in treating chronic ISD in these nonhuman primates (NHPs) [6]. This raises the possibility that cell-mediated native cell mobilization may be a very important contributor to regeneration. As such, the goal of this study was to use quantitative immunohistochemistry [7-9] to compare the effects of autologous cell vs. CXCL12 therapy on mobilization of cells to the urinary sphincter complex of NHPs with chronic urinary sphincter dysfunction.

\section{MATERIALS AND METHODS}

\section{Animal Model}

Studies in these NHPs were approved by the Wake Forest Uni- versity Institutional Animal Care and Use Committee, and were performed in compliance with the Animal Welfare Act and the Guide for the Care and Use of Laboratory Animals. Euthanasia was preformed according to the standards of the American Veterinary Medical Association (AVMA).

\section{Study Design}

Sixteen adult (8-10 years of age) female cynomolgus monkeys (Macaca fascicularis) were used in this study. All 16 monkeys received a bone marrow transplantation with lenti-virus green fluorescent bone marrow cells (BMCs). The 4 study conditions ( $n=4 /$ condition) were: (1) NHPs without sphincter injury (controls) but receiving lenti-M-cherry labeled skMPC injections and BMC transplantations; (2) NHPs with sphincter injury and BMC transplantations, but no treatments; (3) NHPs with sphincter injury, BMC transplantations and sphincter injections of autologous skMPCs; or (4) NHPs with sphincter injury, BMC transplantations and sphincter injections of autologous CXCL12. The timing of BMC and skMPC injections and the sphincter injury procedures are detailed below.

\section{The NHP Model of Urinary Sphincter Injury (ISD)}

Baseline urodynamic measures were obtained in all monkeys. In the monkeys receiving the ISD procedures, the monkeys were sedated with ketamine $10-15 \mathrm{mg} / \mathrm{kg} \mathrm{IM}$ and $1 \%-5 \%$ isoflurane used for induction and maintenance of anesthesia. Monkeys were prepared for aseptic surgery, anesthetized and a lower midline abdominal incision ( $4 \mathrm{~cm}$ in length) made to expose the pelvic area of the abdomen. The distal urinary tract was approached using gentle dissection of connective tissue just ventral to the urinary bladder extending dorsally to the bladder neck and caudally $2 \mathrm{~cm}$ to either side of the rhabdosphincter. The pudendal nerve branches supplying the sphincter (usually three in number) were identified and then selectively electrocauterized - while not damaging the sphincter directly - and then transected [5-8]. Special care was taken not to damage surrounding structures. The abdomen was closed in 2 layers and postoperative support given.

\section{Sequence of Procedures}

Control monkeys received injections of M-cherry labeled skMPCs bone marrow transplantations with green fluorescent protein (GFP)-labeled BMCs at the same time (approximately 3 months after collecting the muscle biopsy and 3 days after suppression of the bone marrow with busulfan -see below). In the 
group of monkeys with the ISD procedure, but no treatments, BMCs were injected 3 months post injury.

In monkeys with ISD and receiving skMPC injections, the skMPCs were collected 6-week post-ISD procedure and injected 3-month post-ISD. The BMCs were collected and labeled 6 days prior to the skMPCs and then injected at the same time as the skMPCs. In monkeys receiving CXCL12, bone marrow samples were taken as described (3-month post-ISD procedure) and both CXCL12 and labeled BMCs injected simultaneously.

\section{skMPC Collection}

A $1-\mathrm{cm}^{3}$ sample of quadriceps muscle was aseptically removed from anesthetized NHP and transported in a wash solution of Dulbecco's Phosphate Buffered Saline (DPBS, HyClone, South Logan, UT, USA) with $1 \%$ antibiotic/antimitotic (HyClone). The tissue was washed 10 minutes $\mathrm{X} 3$ in fresh wash solution with periodic gentle agitation with a final rinse in DBPS. The sample was trimmed of unwanted tissue, weighed, and minced into fragments approximately $0.5 \mathrm{~mm}^{2}$ or less. Digestion media consisting of 2:1 dispase II (Sigma Aldrich, St. Louis, MO, USA): collagenase type I (Worthington, Lakewood, NJ, USA) per ml of basal media (custom designed muscle progenitor cell media, PeproTech, Rocky Hill, NJ, USA) was added to the minced tissue at $1 \mathrm{~mL}$ per $100 \mathrm{mg}$ of tissue. The sample was incubated at $37^{\circ} \mathrm{C}$, $5 \% \mathrm{CO}_{2}$ for 45 minutes. Upon completion, the digestion was terminated using $2 \mathrm{x}$ volume of growth media (PeproTech basal media plus fetal bovine serum (FBS) and custom growth supplements) to digestion media and rigorous pipetting was applied. The suspension was filtered through a $100-\mu$ filter and centrifuged for 5 minutes at 1,500 RPM. The supernatant was aspirated, fresh growth media was added, and spun for a second time. Then the sample was plated on a pretreated collagen-I 100mm culture plate (BD Biocoat, Becton, Dickinson, Franklin Lakes, NJ, USA) and incubated for 24 hours at $37^{\circ} \mathrm{C}, 5 \% \mathrm{CO}_{2}$. The following day, the aspirate was collected and replated on a new pretreated collagen coated plate to reduce fibroblast in the cell culture. The skMPCs were characterized prior to injection by immunocytochemistry and fluorescent antibody cell sorting (FACS) as described previously [10]. It took 4-6 weeks to expand the skMPC cultures up to 5 million cells.

\section{skMPC Transduction and Injection}

Four to 6 weeks following collection of the muscle sample, 5 million skMPCs were transduced with lentivirus (carrying an M-cherry reporter) (Gene Copoeia, Cat LP-EGFP-LV105-0200) (approximately $10 \times 10^{6}$ virus particles/million cells [10 multiplicity of infection]) and Polybrene $(8-\mu \mathrm{g} / \mathrm{mL}$ media), were will be added to the medium for 24 hours. The cells were washed with DPBS 3 times and then cultured in normal media for an additional 24 hours and then observed for labeling efficiency which is usually around $90 \%-95 \%$. The cells were suspended in $2 \mathrm{~mL}$ of sterile PBS and injected directly into the sphincter complex at the 3,6, 9, and 12 oclock position at the level of the external skeletal muscle layer by direct visualization through a laparotomy done aseptically on an anesthetized monkey. We routinely achieve $80 \%$ positive transduction rate without an increase in doubling times. On average, the passage one BMCs were injected 2- to 3-day postcollecion.

\section{Partial Bone Marrow Transplantation}

Autologous BMCs were collected from a bone marrow aspirate and the nucleated cells (which include hematopoietic cells and other progenitor cell populations) were isolated using histopaque. The cells were cultured in QBSF-60 (serum-free hematopoietic stem cell media) with hSCF (50 ng/mL), Flt3-L (50 $\mathrm{ng} / \mathrm{mL}$ ), and PBS, expanded for one passage and given immediately by slow intravenous administration at $15 \times 10^{8}$ cells $/ \mathrm{kg}$ body weight. Forty-eight hours prior to infusion, $15 \times 10^{8} / \mathrm{kg}$ body weight BMCs were transduced with lenti-GFP using techniques described for the skMPCs. We routinely achieve $75 \%-$ $85 \%$ transduction using this method. The BMCs were injected by slow intravenous injection 3 days following a 3 consecutive intravenous injection of busulfan $(1 \mathrm{mg} / \mathrm{kg})$ - used to produce partial bone marrow suppression. BMC characterization was done prior to injection and then a bone marrow biopsy was taken at necropsy and analyzed using quantitative immunocytochemistry as described previously [5].

\section{Collection of Tissues}

Monkeys were euthanized (according to AVMA directives) 6 months following treatment. The vascular system was flushed with 1,000-mL lactated Ringer's solution. The sphincter complex of an adult female cynomolgus monkey is small $(\sim 2 \mathrm{~cm}$ in length). Careful attention was taken to divide this small amount of tissue appropriately and consistently. The urethra was removed from each animal and divided equally into three (proximal, middle and distal) cross sectional segments (approximately $7 \mathrm{~mm}$ in length). Segments used for each measurement were randomized to eliminate positional bias. 


\section{Identifying and Quantifying Labeled Cell Densities Using Standard Immunohistochemistry}

Antibodies used for both skMPC and BMCs were: Skeletal Muscle Actin (1:1,000; ThermoFisher Scientific, Waltham, MA, USA); MyoD (1:100; Pharmingen, San Jose, CA, USA); CD34 (1:100; Miltenyi Biotec, Bergisch Gladbach, Germany); CD44 (1:200; Diaclone, Besançon cedex, France); CD133 1:100; Miltenyi); CD45 1:200; Miltenyi); Oct 4 - following permeablization of the nucleus (1:100; R\&D Systems, Minneapolis, MN, USA), CD117 (1:200; BioLegend, San Diego, CA, USA), anti-GFP
(1:100; Abcam, Cambridge, UK) and anti-M cherry (1:200 Abcam). All immunohistochemistry was done in triplicate with a serum isotype control matched with each antibody. The antibodies used were Pictures were taken with a Leica DM4000B compound microscope with a Retiga 2000RV Q-Imaging camera (Q-Imaging, Surrey, BC, Canada). Sections were blocked using serum free blocking agent (Dako, Carpentaria, CA, USA) to avoid nonspecific binding of primary antibodies. Primary antibody was added on the sections and incubated for 12 hours. FACS was done as described previously by our laboratory [10].
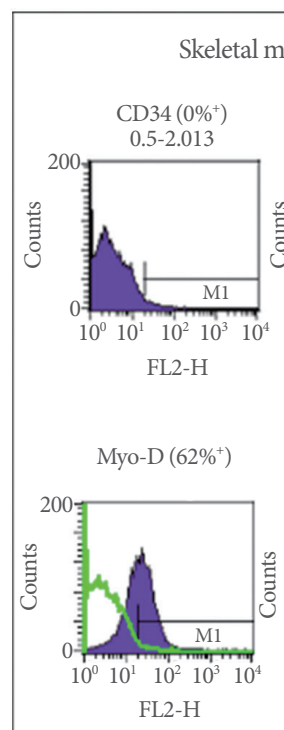

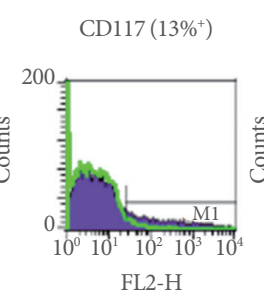

$\operatorname{skM-actin}\left(87 \%^{+}\right)$

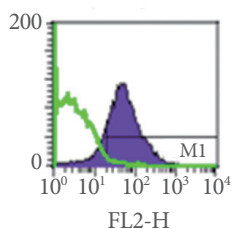

FL2-H

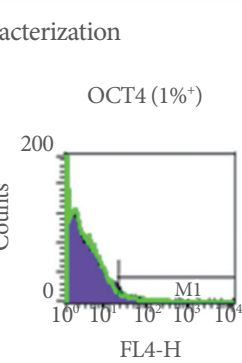

CD44 $(62 \%+)$

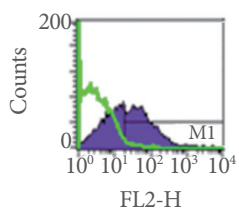

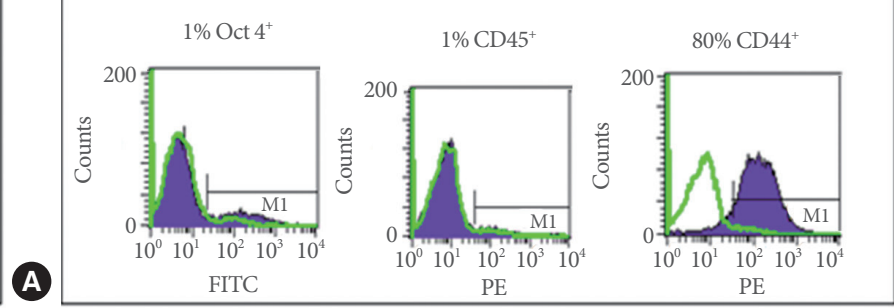

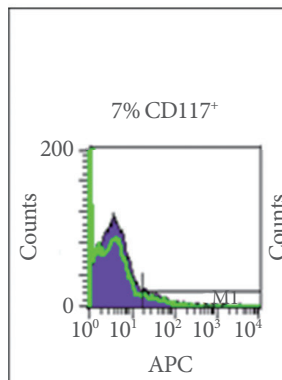

Bone marrow cells
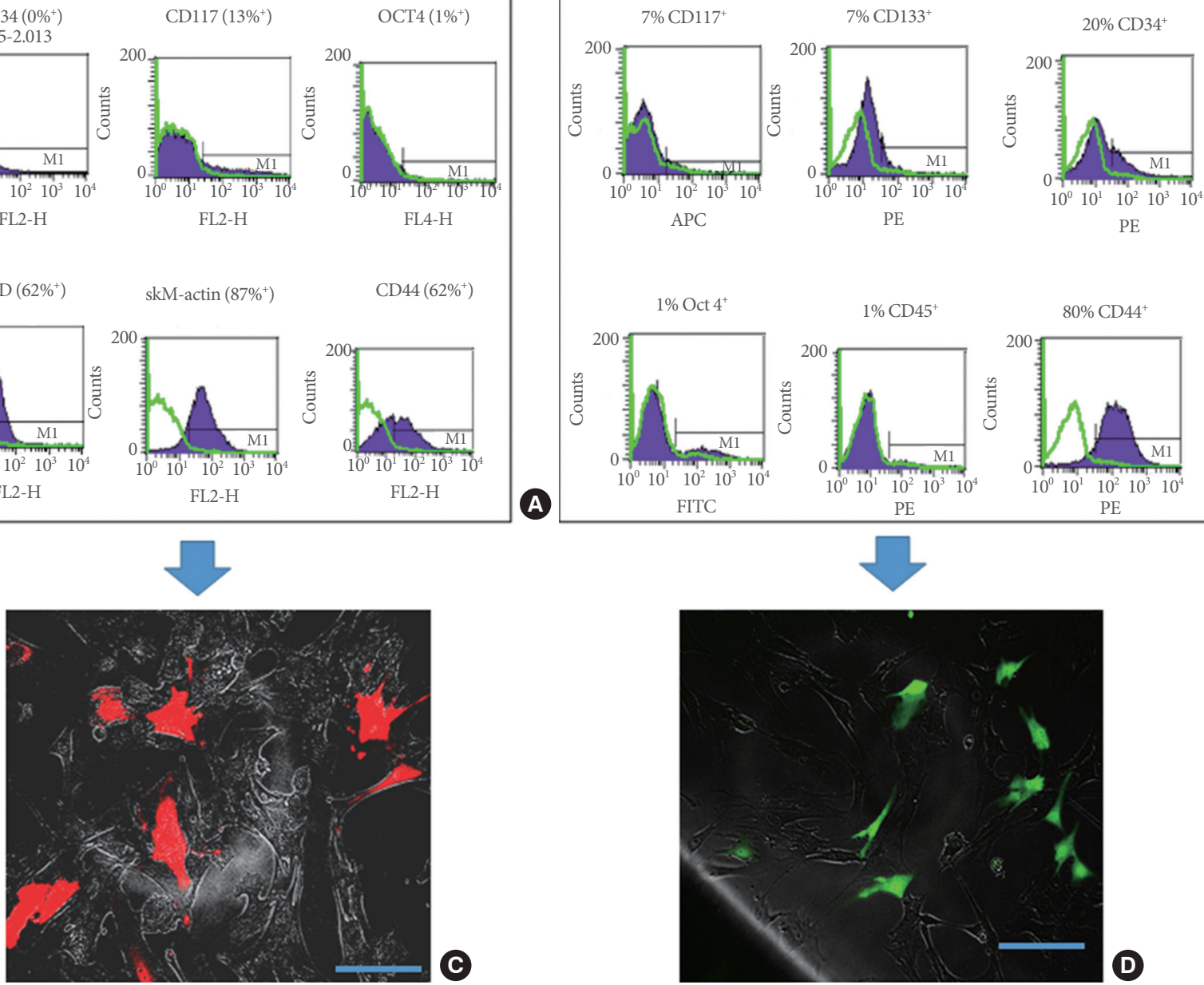

Fig. 1. Characterization of skeletal muscle precursor cells (skMPCs) and bone marrow cells (BMCs) prior to injection. (A) Flow cytometry of skMPCs revealed that passage 2 cells expressed both immature (MyoD) and mature markers (skeletal muscle actin) of skeletal muscle cells as well as general mesenchymal progenitor cells (CD117 and CD44). (C) After lenti-viral transduction an average of $80 \%$ of cells expressed the M-cherry marker. (B) Flow cytometry BMCs revealed that passage 1 cells expressed many markers of progenitor cells (CD117, CD34, CD133, CD44), but were not CD45 positive (thus probably not hematopoietic cells) and not Oct 4 positive (thus probably not stem or early progenitor cells). (D) Similar to the skMPCs, approximately $80 \%$ of cells expressed green fluorescent protein after lenti-viral transduction. Scale bars are $100 \mu \mathrm{m}$. 
For quantifying cell densities in tissues, the tissue area was calculated using Image-Pro AMS 6.0 software (Media Cybernetics, Rockville, MD, USA) as we have done previously in our laboratory [9]. Cell densities were analyzed using computer-assisted morphometric software (Image ProPlus 5.1). Cell densities were determined by overlay of a $10^{2}$ pixel grid with evaluation of each crosshatch for positive or negative staining by a trained observer blinded to treatment. Cell densities were expressed as the percentage of the sphincter tarea occupied by positively stained nucleated cells. Three sections for quantification were taken from the proximal, middle and distal urinary sphincter complex. Four images, representing the four quadrants of the sphincter, we used.

\section{Statistical Approach}

Data are presented as percent of skMPCs and BMCs compared to all nucleated cells in the urinary sphincter complex. These numbers are mean \pm standard error of the mean for skMPCs and BMCs for the treatment groups (no injury, injury, but no treatment, injury+skMPC therapy and injury+CXCL12. These percentages were calculated for the urothelial layer, the lamina propria and the skeletal muscle layer of the sphincter. Student ttest was used and a Bonferroni correction used for multiple comparisons. Statistical significance was set at $\mathrm{P}<0.05$.

\section{RESULTS}

\section{Characterization of skMPCs and BMCs Prior to Injection}

FACS analysis of skMPCs revealed that passage 2 cells ex- pressed both immature (MyoD) and mature (skeletal muscle actin) markers of skeletal muscle cells as well as markers for general mesenchymal progenitor cells (CD117 and CD44) (Fig. 1A). After lenti-viral transduction, an average of $80 \%$ of cells expressed the M-cherry marker (Fig. 1C). Flow cytometry BMCs revealed that passage 1 cells expressed many markers of progenitor cells (CD117, CD34, CD133, CD44), but were not CD45 positive (thus probably not hematopoietic cells) and not Oct 4 positive (thus probably not stem or early progenitor cells) (Fig. 1B). Similar to the skMPCs, approximately $80 \%$ of cells expressed GFP after lenti-viral transduction (Fig. 1D).

\section{Characterization of BMC Cells After Transplantation}

Six months following the injection of GFP-transduced BMCs, bone marrow samples were taken and numerous GFP-green cells could be seen in the bone marrow. They were CD45 negative (Fig. 2A), but CD133, CD117 and CD34 positive (Fig. 2B-D). Thus, labeled cells expressed a variety of progenitor cell markers.

\section{Tissue Analysis}

Fig. 3 depicts immunohistochemistry images of both skMPC (red) and BMC (green) cells in the different areas of the urinary sphincter complex. Both GFP+ and M-cherry+ cells could be seen in all layers of the urinary sphincter complex. The labeled cells were relatively evenly distributed in the urinary sphincter complex except the labeled skMPCs, which were scarce in the urothelium and lamina propria.

Quantitative assessment of the injected muscle precursor versus the BMCs was performed in the various regions of the
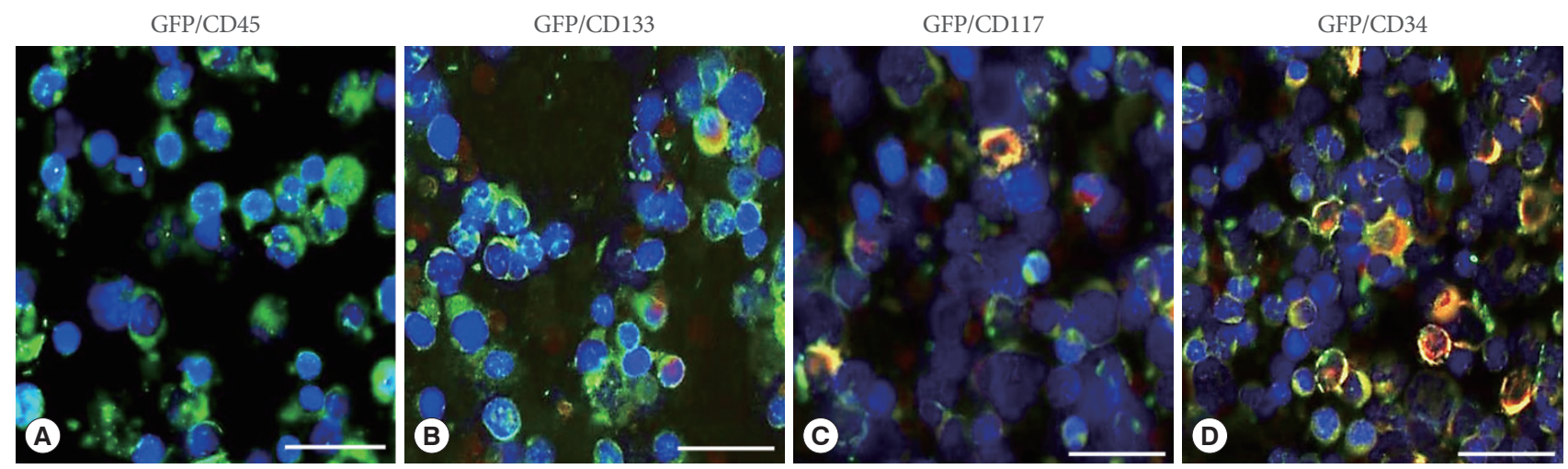

Fig. 2. Characterization of bone marrow cells (BMCs) after transplantation. Six months following the injection of green fluorescent protein (GFP)-transduced BMCs, numerous GFP-green cells could be seen in the bone marrow. (A) When tested for individual marker expression (Texas red staining), they were CD45 negative. (C-E) However, GFP+ cells could be found expressing CD133 (B), CD117 (C), and CD34 (D) (which appear as red or yellow cells). Thus, labeled cells expressed a variety of progenitor cell markers. Scale bars are $200 \mu \mathrm{m}$. 


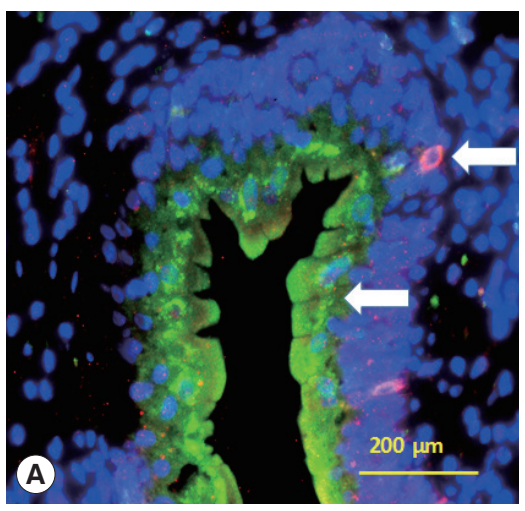

Urothelium

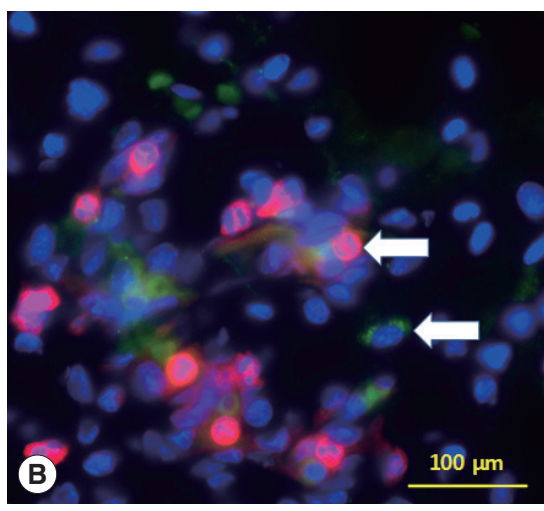

Lamina propria

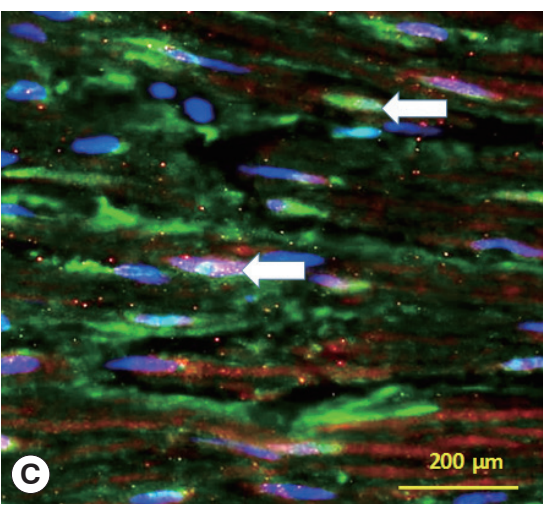

Skeletal muscle layer

Fig. 3. Skeletal muscle precursor cells (skMPCs) and bone marrow cells (BMCs) in the urinary sphincter. Immunohistochemistry images of both injected (red) and native (green) cells in the different areas of the urinary sphincter complex. (A) Green BMCs red injected skMPCs (arrows) in the urothelium. (B) Green BMCs and red skMPCs (arrows) in the lamina propria of the urinary sphincter complex. (C) Green BMCs and injected skMPCs in the skeletal muscle payer of the urinary sphincter complex (arrows).

Table 1. Quantitative assessment of labeled skMPCs and BMCs in the urinary sphincter complex

\begin{tabular}{|c|c|c|c|}
\hline Experimental group & $\begin{array}{c}\text { Skeletal muscle } \\
\text { (\% of nucleated cells) }\end{array}$ & $\begin{array}{c}\text { Smooth muscle } \\
\text { (\% of nucleated cells) }\end{array}$ & $\begin{array}{c}\text { Urothelium } \\
\text { (\% of nucleated cells) }\end{array}$ \\
\hline \multicolumn{4}{|l|}{ Control $^{\mathrm{a})}(\mathrm{n}=4)$} \\
\hline skMPCs & $7 \pm 1$ & $13 \pm 3$ & $7 \pm 2$ \\
\hline BMCs & $3 \pm 4$ & $2 \pm 4$ & $3 \pm 3$ \\
\hline \multicolumn{4}{|l|}{ Chronic injury } \\
\hline $\begin{array}{l}\text { No treatment }{ }^{\mathrm{b})}(\mathrm{n}=4) \\
\text { skMPC treatment }(\mathrm{n}=4)\end{array}$ & $20 \pm 6^{*}$ & $14 \pm 4^{*}$ & $12 \pm 5^{*}$ \\
\hline skMPCs & $12 \pm 3$ & $5 \pm 3$ & $4 \pm 3$ \\
\hline BMCs & $10 \pm 4$ & $8 \pm 3$ & $6 \pm 2$ \\
\hline CXCL12 treatment $(\mathrm{n}=4)$ & $39 \pm 6^{\dagger}$ & $40 \pm 7^{\dagger}$ & $35 \pm 5^{\dagger}$ \\
\hline
\end{tabular}

Values are presented as percent of skeletal muscle precursor cells (skMPCs) and bone marrow cells (BMCs) compared to all nucleated cells in the urinary sphincter complex. These numbers are mean \pm standard error of the mean for skMPCs and BMCs for the treatment groups (no injury, injury, but no treatment, injury+skMPC therapy and injury+CXCL12. These percentages were calculated for the urothelial layer, the lamina propria and the skeletal muscle layer of the sphincter. $\mathrm{n}=4$ for each measure.

${ }^{*} \mathrm{P}<0.05$ vs. control uninjured monkeys. ${ }^{\dagger} \mathrm{P}<0.05$ vs. control and skMPC treated animals. ${ }^{\text {a) }}$ Control: no sphincter injury: skMPC injections and

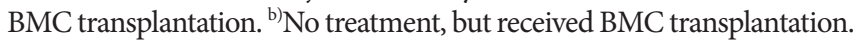

urinary sphincter (Table 1). Sphincter injury resulted in an increased number of labeled BMCs in all layers $(\mathrm{P}<0.05$ vs. noninjured sphincters). We could not assess skMPCs because they were not injected into the injury only group. Injection of skMPCs did not increase the number of skMPCs detected in the sphincter complex and had nonsignificant increases in BMC content. Injection of CXCL12 markedly increased the number of BMCs in the sphincter complex in all three layers $(\mathrm{P}<0.05$ vs. control and injury only groups).

\section{DISCUSSION}

This study reports a method to differentially label both locally injected skMPCs and bone marrow transplanted BMCs with a stable lentivirus promoter and then identify them within urinary sphincter tissue. A comparison between cell injection versus injection of the chemokine CXCL12 indicates that, in the face of chronic urinary sphincter dysfunction, CXCL12 is superior to cell therapy in mobilizing cells to the sphincter. Based on previous work [6], cell mobilization could be an underlying process by which CXCL12 is superior to cell therapy in restoring sphincter structure and function in monkeys with chronic 
urinary sphincter deficiency.

CXCL12 is superior to cell therapy in mobilizing cells to the site of injury. While standard immunohistochemistry (IHC) can measures individual cell densities, multiplex/multispectral analysis of IHC stained tissue provides a method to quantify the cell densities within the sphincter and quantity their expression profiles related to differentiation into sphincter tissues (muscle, matrix, nerve, vessels). We propose that this technology will permit a more comprehensive analysis of the mechanisms by with regenerative therapies affect tissue regeneration.

Several methods have been used to identify and track cells once they are injected, or implanted. What started as tracking cells containing iron particles with standard X-rays has advanced a great deal in recent years. These new tools can allow real-time, quantitative, and long-term monitoring of transplanted cells in the recipient, providing insight on cell migration, distribution, viability, differentiation, and fate, all of which play crucial roles in treatment efficacy. Most cell tracking work has centered on imaging modalities such as MRI, radionuclide imaging, and optical imaging. However, X-ray computed tomography (CT) is an emerging method for cell tracking that has several strengths such as high spatial and temporal resolution, and excellent quantitative capabilities [2-4,11,12].

While providing real time and longitudinal assessments of cell movement, there are drawbacks to these in vivo tracking technologies. They are expensive and not readily available in many centers. They are still less precise than imaging cells and providing spatial relationships of tagged cells within the tissue of interest. Our goal was to differentially label injects and native cells and then quantify their relative in situ contribution to the regenerative processes. For this, careful histologic analysis of relative cell numbers, their expression patterns and 3-dimmensional relationships are needed. This cannot be done with imaging techniques or polymerase chain reaction.

In this study, we used standard IHC. The advantage of lentivirus-transduction is that the label is passed on to daughter cells as the cells divide. Thus, the signal does not diminish significantly with time. Another advantage is that one can target the label (GFP, M-cherry protein) rather than rely on the fluorescence itself (which can vary with time and local tissue conditions) to identify and actually turn it any color one wishes. A disadvantage of standard IHC is that is limits the amount of costaining one can do to further identify the lentivirus-labeled cells. New methods, including multispectral/multiplex imaging [13], can un-mix up to 8 fluorophores on a single slide and thus to quantitatively assess the cellular components of the sphincter [9]. This theoretically permits more extensive characterization of injected and mobilized cells, thus revealing their role in tissue regeneration.

Results of the present study using standard IHC indicate that in the sphincter, the injected skMPCs did not increase in number and actually declined. In contrast, the number of GFP-labeled cells increased in all layers of the urinary sphincter. We have previously reported that injection of skMPC into NHPs with acute ISD restores sphincter structure and function [5-7]. Result of this study indicate that cell mobilization of adjacent or BMCs may contribute more to regeneration than the injected cells.

It was beyond the scope of this study to determine how the injected cells may have stimulated cell mobilization, but their release of chemokines such as CXCL12 have been reported to stimulate cell mobilization, revascularization, reinnervation and regeneration of tissues [6].

The number of monkeys in each group was relatively small and larger numbers may have provided additional clues as to how injected cells stimulate cell mobilization. Most importantly, the role of mobilized cells (their expression patterns, colocalization with vascular and neurogenic markers is essential for longterm successful regeneration of damaged tissue.

In conclusion, this dual labeling methodology now provides us with the tools to accurately assess the relative number of injected cells versus mobilized cells in regenerating tissue and the effects of treatment on these processes. This methodology could be strengthened even further by utilizing new IHC techniques that more accurately assess multiple fluorophores and their quantification. Additionally, these results suggest that after ISD cell mobilization of adjacent or BMCs may contribute more to regeneration than injected cells and could explain why cell therapies have been only modestly effective in treating chronic urinary sphincter deficiency urologic tissues in women.

\section{ACKNOWLEDGEMENTS}

The authors would like to thanks Doug Shankle and Kristina Strumpf for their expert technical assistance with the multiplex/ multispectral imaging study. The datasets used and/or analyzed during the current study are available from the corresponding author on reasonable request. All data generated or analyzed during this study are included in this published article. 


\section{AUTHOR CONTRIBUTION STATEMENT}

- Full access to all the data in the study and takes responsibility for the integrity of the data and the accuracy of the data analysis: JKW, SM, IS, SSL, KEA

- Study concept and design: JKW, SL, KEA

- Acquisition of data: $J K W, S L, K E A$

- Analysis and interpretation of data: JKW, SM, IS, SSL, KEA

- Drafting of the manuscript: JKW, SM, IS, SSL, KEA

- Critical revision of the manuscript for important intellectual content: JKW, SM, IS, SSL, KEA

- Statistical analysis: $J K W$

- Obtained funding: JKW, KEA

- Administrative, technical, or material support: JKW, SM, IS, SSL, KEA

-Study supervision: JKW, SL, KEA

\section{REFERENCES}

1. Williams JK, Dean A, Lankford S, Criswell T, Badlani G, Andersson KE. Determinates of muscle precursor cell therapy efficacy in a nonhuman primate model of intrinsic urinary sphincter deficiency. Stem Cell Res Ther 2017;8:1.

2. Liu S, Zhou J, Zhang X, Liu Y, Chen J, Hu B, et al. Strategies to optimize adult stem cell therapy for tissue regeneration. Int J Mol Sci 2016;17.

3. Syková E, Jendelová P. Magnetic resonance tracking of transplanted stem cells in rat brain and spinal cord. Neurodegener Dis 2006;3:627.

4. Jurgielewicz P, Harmsen S, Wei E, Bachmann MH, Ting R, Aras O. New imaging probes to track cell fate: reporter genes in stem cell research. Cell Mol Life Sci 2017;74:4455-69.
5. Williams JK, Badlani G, Dean A, Lankford S, Poppante K, Criswell $\mathrm{T}$, et al. Local versus intravenous injections of skeletal muscle precursor cells in nonhuman primates with acute or chronic intrinsic urinary sphincter deficiency. Stem Cell Res Ther 2016;7:147.

6. Williams JK, Dean A, Badra S, Lankford S, Poppante K, Badlani G, et al. Cell versus chemokine therapy in a nonhuman primate model of chronic intrinsic urinary sphincter deficiency. J Urol 2016;196:180915.

7. Badra S, Andersson KE, Dean A, Mourad S, Williams JK. Longterm structural and functional effects of autologous muscle precursor cell therapy in a nonhuman primate model of urinary sphincter deficiency. J Urol 2013;190:1938-45.

8. Badra S, Andersson KE, Dean A, Mourad S, Williams JK. A nonhuman primate model of stable urinary sphincter deficiency. J Urol 2013;189:1967-74.

9. Soler R, Vianello A, Füllhase C, Wang Z, Atala A, Soker S, et al. Vascular therapy for radiation cystitis. Neurourol Urodyn 2011;30:42834.

10. Williams JK, Baptista PM, Daunais JB, Szeliga KT, Friedman DP, Soker $S$. The effects of ethanol consumption on vasculogenesis potential in nonhuman primates. Alcohol Clin Exp Res 2008;32:15561.

11. Kim J, Chhour P, Hsu J, Litt HI, Ferrari VA, Popovtzer R, et al. Use of nanoparticle contrast agents for cell tracking with computed tomography. Bioconjug Chem 2017;28:1581-97.

12. Thébaud NB, Aussel A, Siadous R, Toutain J, Bareille R, Montembault $\mathrm{A}$, et al. Labeling and qualification of endothelial progenitor cells for tracking in tissue engineering: an in vitro study. Int J Artif Organs 2015;38:224-32.

13. Spaeth EL, Booth CM, Marini FC. Quantitative multispectral analysis following fluorescent tissue transplant for visualization of cell origins, types, and interactions. J Vis Exp 2013:e50385. 